\title{
Heat transfer in solid methyl alcohol
}

\author{
O.A. Korolyuk, A.I. Krivchikov, I.V. Sharapova, and O.O. Romantsova \\ B. Verkin Institute for Low Temperature Physics and Engineering of the National Academy of Sciences of Ukraine \\ 47 Lenin Ave., Kharkov 61103, Ukraine \\ E-mail:korolyuk@ilt.kharkov.ua
}

Received January 26, 2009

\begin{abstract}
Thermal conductivity coefficient $\kappa(T)$ of two crystalline (orientationally-ordered and orientationally-disordered) phases of pure methanol (at temperatures from $2 \mathrm{~K}$ to $T_{m}, T_{m}$ is the melting temperature), $\mathrm{CH}_{3} \mathrm{OH}+6.6 \% \mathrm{H}_{2} \mathrm{O}$ glass from $2 \mathrm{~K}$ to $T_{g}, T_{g}$ is the glass transition temperature and a supercooled liquid from $T_{g}$ to $120 \mathrm{~K}$ has been measured under equilibrium vapor pressure. The dependence $\kappa(T)$ is described approximately as a sum of two contributions: $\kappa_{\mathrm{I}}(T)$ describing heat transport by acoustic phonons and $\kappa_{\mathrm{II}}(T)$ - by localized high-frequency excitations. The temperature dependences of the thermal conductivity of primary monoatomic alcohols $\mathrm{CH}_{3} \mathrm{OH}, \mathrm{C}_{2} \mathrm{H}_{5} \mathrm{OH}$, and $\mathrm{C}_{3} \mathrm{H}_{7} \mathrm{OH}$ in the glass state have been compared. Different mechanisms of phonon scattering in the crystalline phases and glass have been analyzed. The $\kappa_{\mathrm{II}}(T)$ has been calculated within the Cahill-Pohl model. There is an anomaly of the thermal conductivity of the glass state near $T_{g}$ (a smeared minimum in the $\kappa(T)$ - curve).
\end{abstract}

PACS: 66.70.-f Nonelectronic thermal conduction and heat-pulse propagation in solids; thermal waves;

63.20.-e Phonons in crystal lattices;

63.50. $-\mathbf{x}$ Vibrational states in disordered systems.

Keywords: heat transport, acoustic phonons, thermal conductivity, monoatomic alcohols.

\section{Introduction}

Methanol is a monoatomic primary alcohol. The single methyl group $\mathrm{CH}_{3}$ of the methanol molecule is bound to the hydroxyl group $\mathrm{OH}$. Among the primary alcohols, methanol has the shortest and most mobile molecule, which makes it a suitable object for modeling the properties of alcohols having more complex molecules [1-6]. The simple structure of the methanol molecule shows up most obviously in its properties [2-12]. In contrast to water ice which, under normal conditions, is an associated substance with strong tetragonally directed cooperative $\mathrm{H}$ bonds, monoatomic alcohols in the condensed state are viewed as associated objects with moderate $\mathrm{H}$ bonds and a chain-like structure $[1,4]$.

The effects of the cooperative hydrogen bond in different properties of alcohols decreases as the number of carbon atoms in the molecule increases. At varying temperature and pressure, methanol displays more equilibrium phases than ethanol [4-6,13]. Polymorphism of the crystalline phases of methanol is governed by its $\mathrm{H}$ bonds which are stronger than the dispersive molecular interactions and by its simpler molecular structure in comparison to ethanol. It is found in the previous studies [14-16] of thermal conductivity of alcohols (ethanol and isomers of propyl alcohol) in different polymorphous states that ethanol has similar temperature dependence of thermal conductivity (especially at low temperatures) in the phases of a metastable orientationally disordered crystal and a glass (structurally disordered solid). In the propyl alcohols (1-propyl alcohol and 2-propyl alcohol) a strong isomeric effect in the temperature dependence of thermal conductivity was observed.

Under equilibrium vapor pressure and by lowering temperature, methanol crystallizes at $T_{m}=175.37 \mathrm{~K}$ into the orientationally disordered high-temperature state ( $\beta$-phase). Upon the further decrease of temperature, a solid-solid transformation into the low-temperature orientationally-ordered state ( $\alpha$-phase) occurs at $T_{\alpha-\beta}=$ $=157.4 \mathrm{~K}$ [13]. Their space groups are $P 2{ }_{1} 2_{1} 2_{1}$ for $\alpha$-phase and $C m c m$-phase [13]. Unlike the situation in ethanol and propanol, fast cooling of the liquid phase hinders the glass transition in methanol. It proceeds more prominently when vapor is condensed onto a cold substrate below $T_{g}=103.4 \mathrm{~K}[8,17]$. Poor glass-forming ability of this substance is caused by the structure of its liquid and crystalline phases which consist of zigzag chains of alternating H-bonded molecules. The glass 
phase of methanol can be obtained by adding a small quantity of water $\left(\sim 6.5 \mathrm{~mol} . \% \mathrm{H}_{2} \mathrm{O}\right)$ to it [18]. The glass transition in methanol with water admixture occurs in rather wide temperature interval $T_{g}=100-120 \mathrm{~K}$ [19].

In this study the thermal conductivity has been measured, for the first time, on pure methanol in different states - equilibrium crystalline phases from 2 to $175 \mathrm{~K}$, glass from $2 \mathrm{~K}$ to $T_{g}$, and supercooled liquid from $T_{g}$ to $120 \mathrm{~K}$ of methanol with water admixture. The measurements were made under equilibrium vapor pressure using the method of steady-state linear heat flow.

\section{Experiment and discussion}

The thermal conductivity of different phases of solid methanol was measured under equilibrium vapor pressure in a set-up described earlier [20] using the steady-state potentiometric method. The container for the sample [20] was a stainless steel tube. Two copper wires $1 \mathrm{~mm}$ in diameter were placed across the container perpendicular to its axis, which permitted measurement of the average temperature in the isothermal planes across the sample. At the outer surface of the container, copper sockets were soldered to the wires to hold temperature sensors. The liquid methanol sample was admitted into the container of the measuring cell under ${ }^{4} \mathrm{He}$ gas. The helium gas was used to improve the heat exchange between the sample and the container. The container with the sample was vacuum-tight closed by a copper cap with an indium O-ring. A heater was mounted on the container cap to generate a downward heat flow along the sample.

The thermal conductivity of pure methanol and its solution with distilled water was measured. According to chromatographic analysis, the water content in the pure methanol was less than $0.2 \% \mathrm{H}_{2} \mathrm{O}$. The water-methanol solution contained $6.6 \mathrm{~mol} . \% \mathrm{H}_{2} \mathrm{O}$.

The samples in the glass and crystalline phases ( $\alpha$ and $\beta$ ) were prepared using the techniques applied to ethanol $[14,15]$. The measurements were performed at gradually decreasing temperature. After reaching the lowest temperature point the measurement was continued at increasing temperature. Crystalline sample was grown in the measuring container during slow cooling the liquid slightly below $T_{m}$. Thermal conductivity was measured on crystalline samples of pure methanol and methanol with $6.6 \% \mathrm{H}_{2} \mathrm{O}$. The water impurity had practically no effect on the magnitude of the thermal conductivity of methanol.

The glass-state sample of methanol with $\mathrm{H}_{2} \mathrm{O}$ admixture was created by very fast (above $50 \mathrm{~K} \cdot \mathrm{min}^{-1}$ ) cooling the liquid to the temperature of liquid $\mathrm{N}_{2}$ (boiling at normal pressure). By heating above $T_{g}$ the glass sample transformed into a supercooled liquid. Upon the further increase of temperature, above $T \approx 121 \mathrm{~K}$ the supercooled liquid crystallized spontaneously, thermal conductivity of the sample increasing abruptly.

The experimental temperature dependence of the thermal conductivity $\kappa(T)$ measured on methanol crystals ( $\alpha$ - and $\beta$-phases), glass, and the supercooled liquid methanol with $6.6 \% \mathrm{H}_{2} \mathrm{O}$ are shown in Fig. 1 along with the temperatures of glass $\left(T_{g}\right)$ and orientational phase $\left(T_{\alpha-\beta}\right)$ transitions.

The dependence $\kappa(T)$ of the crystalline sample exhibits some interesting features. The thermal conductivity of the $\beta$-phase (orientationally disordered crystal) is independent of temperature (see Fig. 1,b). This agrees with the fact that methanol glass has many structural features in common with the $\beta$-phase crystal $[2,8,7]$. In the orientati-
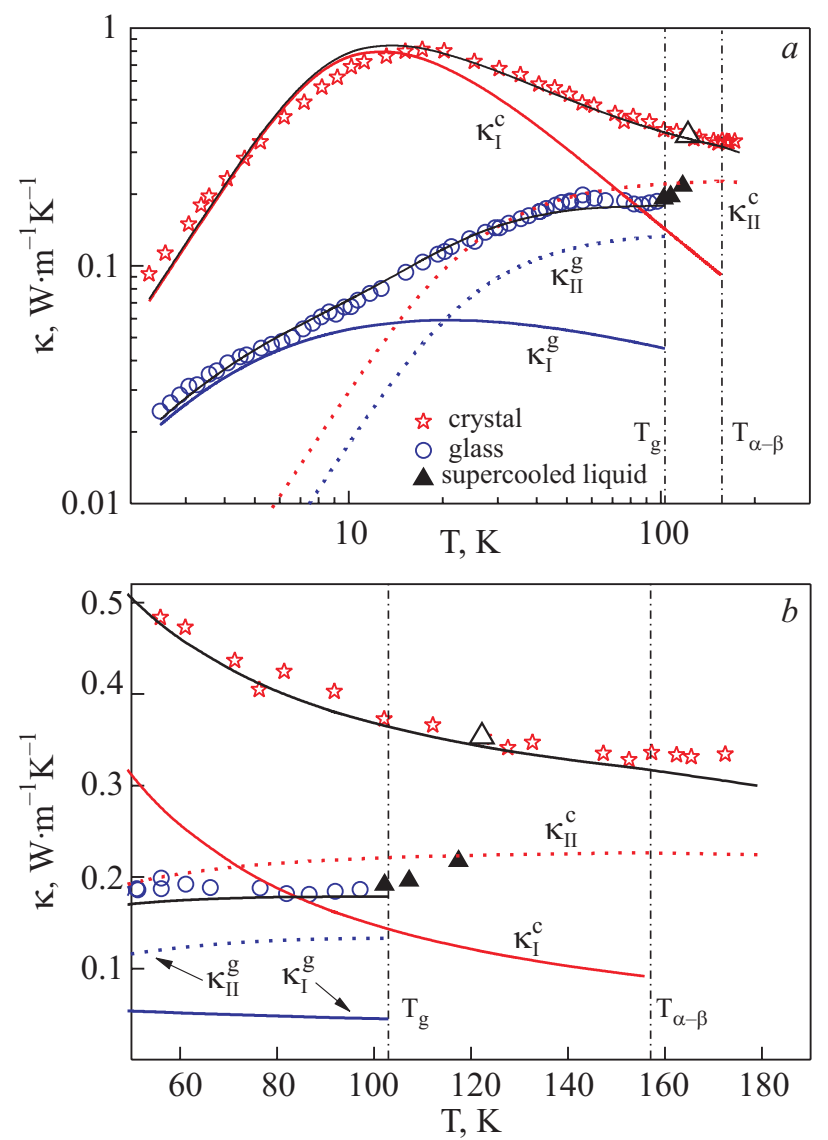

Fig. 1. Temperature dependence of the thermal conductivity in the $\alpha$ - and $\beta$-phases ( $\forall$ ) of pure methanol and glass $(O)$ and in the supercooled liquid $(\boldsymbol{\Delta})$ of methanol with $6.6 \% \mathrm{H}_{2} \mathrm{O}$. Thermal conductivity of the sample after crystallization from the supercooled liquid at $T=121 \mathrm{~K}(\triangle)$. Dashed straight lines are $T_{g}$ and $T_{\alpha-\beta}$. Theoretical calculation of contributions of thermal conductivity for $\alpha$-phase and the glass state: $\kappa_{\mathrm{I}}(T)$ (solid lines) refers to the propagating acoustic phonons; $\kappa_{\mathrm{II}}(T)(\operatorname{dot}$ lines) accounts for the localized short-wavelength modes. Solid lines are the sum $\kappa=\kappa_{\mathrm{I}}^{j}+\kappa_{\mathrm{II}}^{j}$, where $j=c$ or $g$ for $\alpha$-phase and the glass state respectively. The results are presented on double logarithmic scale in a wide interval of temperatures $(a)$ and in the usual scale in the interval $T>60 \mathrm{~K}(b)$. 
onally ordered $\alpha$-phase, $\kappa(T)$ increases at lowering temperature, passes through a broad maximum and then decreases, i.e., the curve has a bell-like shape, typical for an orientationally ordered molecular crystal. Thermal conductivity of the glass sample is distinctly different: $\kappa(T)$ increases with temperature, has a small kink near $T=7 \mathrm{~K}$, goes through a smeared maximum at $T \approx 55 \mathrm{~K}$, then decreases down to a broad minimum and starts to increase again (in the supercooled liquid state). The highest value of $d \kappa(T) / d T$ is observed below $4 \mathrm{~K}$. The broad minimum in the $\kappa(T)$ curve is an anomalous feature of the thermal conductivity of glass [21] (see Fig. 1,b). Thermal conductivity of methanol- $\mathrm{H}_{2} \mathrm{O}$ sample crystallized at $T>121 \mathrm{~K}$ coincides, within the experimental error, with $\kappa(T)$ of the sample prepared by crystallizing the pure methanol at $T \approx T_{m}$.

The behavior of $\kappa(T)$ of the methanol glass is similar to that of the glasses of ethanol and 1-propanol [14,15] (see Fig. 2). In comparison with other alcohols, $\kappa(T)$ of methanol glass increases most rapidly with temperature up to a broad maximum. The highest values of $\kappa(T)$ in methanol are close to those in ethanol and 1-propanol. It is especially interesting that the anomaly in the $\kappa(T)$ curve shows up in the interval $50-120 \mathrm{~K}$. The anomalous behavior of the thermal conductivity can be approximately described by the dependence $\kappa(T)=a T^{-1}+b+c T\left(a=2.25 \mathrm{~W} \cdot \mathrm{m}^{-1}\right.$; $b=0.125 \mathrm{~W} \cdot \mathrm{m}^{-1} \mathrm{~K}^{-1} ; c=0.00043 \mathrm{~W} \cdot \mathrm{m}^{-1} \mathrm{~K}^{-2}$ ) but its origin is not yet fully understood. The results of this study furnish convincing evidence that the anomaly of $\kappa(T)$ is a typical characteristic of the curve describing thermal conductivity of glass state of a solid alcohol.

The temperature dependence of the thermal conductivity of the orientationally-ordered phase and glass state can be interpreted assuming that $\kappa(T)$ consists of two components [22-29]:

$$
\kappa(T)=\kappa_{\mathrm{I}}(T)+\kappa_{\mathrm{II}}(T) .
$$

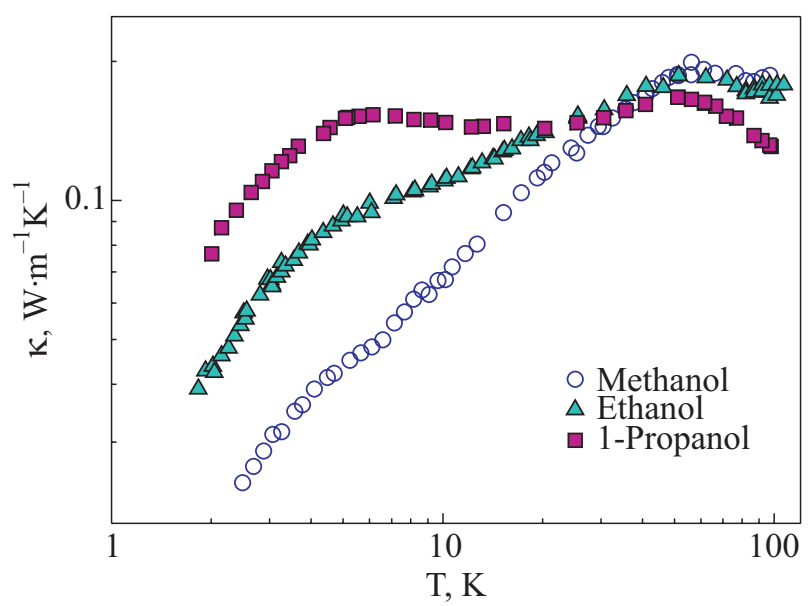

Fig. 2. Temperature dependences of the thermal conductivity of pure 1-propanol [16] ( $\mathbf{\square})$, ethanol $[14,15](\mathbf{\Delta})$, and methanol with $6.6 \% \mathrm{H}_{2} \mathrm{O}$ in glass state (this work) $(\mathrm{O})$.
The component $\kappa_{\mathrm{I}}(T)$ refers to the propagating phonons whose mean free path is longer than the phonon half-wavelength. The phonons are the main heat-transferring particles. The component $\kappa_{\mathrm{II}}(T)$ accounts for the localized short-wavelength vibrational modes, or phonons with the mean free path equal to the phonon half-wavelength. This approximation was used before to interpret $\kappa(T)$ of ethanol glasses [14] and isomers of propanol [15].

The contribution $\kappa_{\mathrm{I}}(T)$ can be calculated within the Debye-Peierls time-relaxation model. In the $\kappa_{\mathrm{I}}(T)$ case of the orientationally-ordered crystalline phase (at the temperature to the right of the phonon maximum) the phonon-rotational and phonon-phonon scattering mechanisms dominate. They are effectively described by the Umklapp processes. The total inverse relaxation time (rate) of phonons is assumed to obey the Matthiesen rule and, therefore, can be expressed as a sum of rates representing different processes leading to phonon scattering. For an ordered crystal, it is expected that the dominant mechanisms able to scatter heat-carrying phonons will concern anharmonic Umklapp processes with the rate $\tau_{U}^{-1}$, and scattering by dislocations $-\tau_{\text {dis }}^{-1}$. Relevant expressions for the scattering processes are given by:

$$
\begin{gathered}
\tau_{U}^{-1}(\omega, T)=B \omega^{2} T \exp \left[-E_{U} / T\right], \\
\tau_{\text {dis }}^{-1}(\omega, T)=D_{\mathrm{dis}} \omega,
\end{gathered}
$$

where $\omega$ is the phonon frequency, $B$ is the frequency-factor, $E_{U}$ is activation energy for the $U$-processes, and $D_{\text {dis }}$ is the dislocation scattering strength.

The temperature dependence of the contribution $\kappa_{\mathrm{I}}(T)$ to the thermal conductivity of glass can be described phenomenologically using the soft potential model (SPM) [30] which portrays phonon scattering as mainly caused by low-energy excitations of a strongly anharmonic ensemble of particles. The scattering rate of acoustic phonons in a disordered system $\tau_{G}^{-1}$ is given by a sum of three terms describing scattering by the tunnel states, classical relaxors and soft quasiharmonic vibrations, and reads:

$$
\begin{gathered}
\tau_{G}^{-1}=\bar{C} \pi \omega\left[\tanh \left(\frac{\hbar \omega}{2 k_{B} T}\right)+\left(\frac{T}{W}\right)^{3 / 4} \ln ^{-1 / 4}\left(\frac{1}{\omega \tau_{0}}\right)+\right. \\
\left.+\frac{1}{6 \sqrt{2}}\left(\frac{\hbar \omega}{W}\right)^{3}\right] .
\end{gathered}
$$

Here $\tau_{0}$ is the inverse of an attempt frequency and is of the order of $10^{-13} \mathrm{~s}$, which account, made of the sound wave frequencies $(10-100 \mathrm{GH})$, yields a logarithmic factor $\ln ^{-1 / 4}\left(1 / \omega \tau_{0}\right) \approx 0.7$. The most relevant parameters are: the dimensionless $\bar{C}$ parameter and the characteristic energy $W$. 
The component $\kappa_{\mathrm{II}}(T)$ refers to the heat transfer by localized harmonic short-wavelength vibrational modes, or by acoustic phonons with the mean free path equal to the phonon half-wavelength. The simplest description of $\kappa_{\mathrm{II}}(T)$ is provided by the phenomenological Cahill-Pohl model [31] which was proposed to interpret the thermal conductivity of amorphous solids at high temperatures. The model accounts adequately for the features of the isochoric high-temperature thermal conductivity of molecular crystals in the orientationally-ordered phase [32]. According to the model, the thermal conductivity is:

$$
\kappa_{\mathrm{II}}=\left(\frac{\pi}{6}\right)^{1 / 3} \kappa_{B} n^{2 / 3} \sum_{i} v_{i}\left\{\left(\frac{T}{\Theta_{i}}\right)^{2} \int_{0}^{\Theta / T} \frac{x^{3} e^{x}}{\left(e^{x}-1\right)^{2}} d x\right\},
$$

where $k_{B}$ is the Boltzmann constant, $n$ is the number of atoms per unit volume; summation is over three vibrational modes with the sound velocities $v_{i}, i$ is the index of summation over the phonon mode polarizations, $\Theta_{i}$ is the Debye temperature for each polarization, $x=\hbar \omega /\left(k_{B} T\right)$. In the Debye approximation for the phonon spectrum of an isotropic solid (the difference between the polarizations of the phonon modes is disregarded) $\kappa_{\mathrm{II}}(T)$ becomes:

$$
\kappa_{\mathrm{II}}=3 F\left(\frac{1}{36 \pi}\right)^{1 / 3}\left(\frac{\rho N_{A}}{M}\right)^{1 / 3} \frac{\kappa_{B}^{2} T^{2}}{\hbar \Theta} \int_{0}^{\Theta / T} \frac{x^{3} \mathrm{e}^{x}}{\left(e^{x}-1\right)^{2}} d x,
$$

where $\rho$ is the density, $M$ is the molar mass, $N_{\mathrm{A}}$ is the Avogadro number, $\Theta$ is the averaged Debye temperature, $F$ is the dimensionless fitting parameter allowing effectively for the intensity of the heat transfer by localized excitations, including the rotational ones. Note that in the orientationally disordered phase $\kappa(T)$ is determined by localized states of different origin.

The thermal conductivity of methanol was calculated using: $M=32.04 \mathrm{~g} / \mathrm{mol} ; \rho=1015 \mathrm{~kg} / \mathrm{m}^{3}$ [13]; the mean sound velocity $v=1400 \mathrm{~m} / \mathrm{s}$ was calculated using the data for the longitudinal sound velocity [33]; $\Theta=106 \mathrm{~K}$ was calculated from the $v$-value.

Table 1 gives the fitting parameters obtained by comparing experimental results and calculated curves.

Table 1. Parameters obtained by fitting theoretical and experimental data

\begin{tabular}{c|c|c|c|c|c|c}
\hline \hline & $B, \mathrm{~s} / \mathrm{K}$ & $E_{U}, \mathrm{~K}$ & $D_{\mathrm{dis}}$ & $\bar{C}$ & $\mathrm{~W}, \mathrm{~K}$ & $F$ \\
\hline \hline$\alpha$-phase & $5.3 \cdot 10^{-16}$ & 18 & $4.6 \cdot 10^{-3}$ & - & - & $4.8 \pm 0.2$ \\
$\beta$-phase & 0 & - & 0 & - & - & $7.0 \pm 0.2$ \\
glass & 0 & - & 0 & $2.9 \cdot 10^{-3}$ & 5 & $2.9 \pm 0.2$ \\
\hline \hline
\end{tabular}

The curves in Fig. 1 are the dependences $\kappa_{\mathrm{I}}(T), \kappa_{\mathrm{II}}(T)$, and $\kappa(T)$ calculated for $\alpha$-phase and the glass state. At temperatures near and to the left of the $\kappa(T)$ maximum, the thermal conductivity of the $\alpha$-phase of solid methanol is mainly determined by $\kappa_{\mathrm{I}}(T)$. Above the maximum and up to $T_{\alpha-\beta}$, the share of $\kappa_{\mathrm{II}}(T)$ increases with temperature. No $\kappa_{\mathrm{I}}(T)$ is observed in the $\beta$-phase. Similar behavior of $\kappa_{\mathrm{I}}(T)$ and $\kappa_{\mathrm{II}}(T)$ contributions to the total thermal conductivity is observed in the glass state of methanol with water admixture: $\kappa_{\mathrm{I}}(T)$ determines the thermal conductivity at temperatures to the left of the kink at $T<7 \mathrm{~K}$. At $T>10 \mathrm{~K}$ the component $\kappa_{\mathrm{II}}(T)$ starts to increase rapidly with temperature. The theoretical curve offered by this model is inadequate to explain the smeared minimum of $\kappa(T)$ near $T=80 \mathrm{~K}$ in the glass state. The division of $\kappa(T)$ into two components permits a satisfactory description of thermal conductivities in a substance capable of developing different molecular structures with different degrees of orientational and translational ordering. McGaughey and Kaviany [28] accept it as a universal theoretical tool. The behavior and magnitude of the contribution $\kappa_{\mathrm{II}}(T)$ to the thermal conductivity of an orientationally ordered crystal $\kappa_{I I}^{c}$ is similar to that in the glass state $\kappa_{I I}^{g}$, which prompts the conclusion that the contribution $\kappa_{\mathrm{II}}$ is practically independent of the substance state (glass or orientationally-ordered crystal). When the $\alpha-\beta$ phase transition occurs, it is accompanied with a volume jump $\Delta V / V \approx 4 \%$ [7]. However, there is no jump (within the experimental error) of the thermal conductivity (see Fig. 1,b). The reason may be that in the region below $T_{\alpha-\beta}$ the thermal conductivity is determined not by the phonon contribution $\kappa_{I}$ sensitive to volume variations but by the contribution $\kappa_{\mathrm{II}}$ from localized vibrational modes, and $\kappa_{I I}$ is only slightly sensitive to changes in the volume [34]. The contribution $\kappa_{\text {II }}$ referring to the heat transport by localized states is independent of the molecular structure of methanol and is determined primary by the increasing intensity of rotation of the methyl and hydroxyl groups of the molecule and their cooperative rotational hopping of the molecules at increasing temperature. The coupling of acoustic phonons and localized excitations becomes most efficient above $10 \mathrm{~K}$ and increases with temperature [18,19].

Summing, the thermal conductivity of methanol has been measured for the first time on samples in different states - equilibrium crystalline phases (from $2 \mathrm{~K}$ to $T_{m}$ ), glass state (from $2 \mathrm{~K}$ to $T_{g}$ ) and supercooled liquid (from $T_{g}$ to $120 \mathrm{~K}$ ) of methanol with water admixture. It is shown that in the solid states the thermal conductivity $\kappa(T)$ is a sum of two contributions: $\kappa_{\mathrm{I}}(T)$ corresponds to propagating phonons which are the main heat-transporting particles and are dependent on the translational and orientational ordering of molecules. The component $\kappa_{\mathrm{II}}(T)$ accounts for the heat transport by localized excitations. $\kappa_{\mathrm{II}}$ is almost independent of the methanol state (glass or an orientationally-ordered crystal). An anomalous feature has been detected in the thermal conductivity 
of the methanol glass: a smeared minimum in the curve $\kappa(T)$ near the glass transition temperature. This behavior of $\kappa(T)$ is similar to that encountered in ethanol glass and two isomers of propanol [21].

The new results and a detailed analysis of the thermal conductivity of monoatomic alcohols both in the metastable states and in thermodynamically equilibrium phases are planned to be published in a subsequent paper.

The authors are sincerely grateful to B.Ya. Gorodilov, Prof. V.G. Manzhelii and Prof. F.J. Bermejo for helpful discussions and interest in this study. The investigations is made on the competition terms for joint projects of NAS of Ukraine and Russian Foundation for Fundamental Research (Agreement N 9-2008, Subject: «Collective processes in metastable molecular solids»).

1. D.C. Steytler, J.C. Dore, and D.C. Montague, J. Non Cryst. Solids 74, 303 (1985).

2. T. Doba, K.U. Ingold, A.H. Reddoch, W. Siebrand, and T.A. Wildman, J. Chem. Phys. 86, 6622 (1987).

3. J.M. Brown, L.J. Slutsky, K.A. Nelson, and L.-T. Cheng, Science 241, 4861, 65 (1988).

4. B.H. Torrie, S.-X. Weng, and B.M. Powell, Mol. Phys. 67, 575 (1989).

5. S. Lucas, D. Ferry, B. Demirdjian, and J. Suzanne, J. Phys. Chem. B109, 18103 (2005).

6. E.L. Gromnitskaya, O.V. Stal'gorova, O.F. Yagafarov, V.V. Brazhkin, A.G. Lyapin, and S.V. Popova, JETP Lett. 80, 597 (2004).

7. D.W. Davidson, Can. J. Chem. 34, 1243 (1956).

8. M. Sugisaki, H. Suga, and S. Seki, Bull. Chem. Soc. Jpn. 41, 2586 (1968).

9. H.G. Carlson and Edgar F. Westrum Jr., J. Chem. Phys. 54, 1464 (1971).

10. F.J. Bermejo, F. Batallan, E. Enciso, M. Garcia-Hernandes, J. Alonso, and J.L. Martinez, Europhys. Lett. 12, 129 (1990).

11. F.J. Bermejo, F. Batallán, J.L. Martínez, M. Garcна Hernández, and E. Enciso, J. Phys.: Cond. Matt. 2, 6659 (1990).

12. F.J. Bermejo, J.L. Martinez, M. Garcia-Hernandez, D. Martin, F.J. Mompean, J. Alonso, and W.S. Howells, Europhys. Lett. 15, 509 (1991).

13. B.H. Torrie, O.S. Binbrek, M. Strauss, and I.P. Swainson, J. Solid State Chem. 166, 415 (2002).

14. A.I. Krivchikov, A.N. Yushchenko, V.G. Manzhelii, O.A. Korolyuk, F.J. Bermejo, R. Fernandez-Perea, C. Cabrillo, and M.A. Gonzalez, Phys. Rev. B74, 060201 (2006).

15. F.J. Bermejo, R. Fernandez-Perea, C. Cabrillo, A.I. Krivchikov, A.N. Yushchenko, O.A. Korolyuk, V.G. Manzhelii, M.A. Gonzalez, and M. Jimenez-Ruiz, Fiz. Nizk. Temp. 33, 790 (2007) [Low Temp. Phys. 33, 606 (2007)].

16. A.I. Krivchikov, A.N. Yushchenko, O.A. Korolyuk, F.J. Bermejo, R. Fernandez-Perea, I. Bustinduy, and M.A. Gonzalez, Phys. Rev. B77, 024202 (2008).
17. Susan M. Dounce, Julia Mundy, and Hai-Lung Dai, J. Chem. Phys. 126, 191111 (2007).

18. F.J. Bermejo, D. Martin, J.L. Martínez, F. Batallan, M. García-Hernández, and F.J. Mompean, Phys. Lett. A150, 201 (1990).

19. F.J. Bermejo, M. García Hernández, J.L. Martínez, A. Criado, and W.S. Howells, J. Chem. Phys. 96, 7696 (1992); F.J. Bermejo, J. Alonso, A. Criado, F.J. Mompean, J.L. Martinez, M. Garcia-Hernandez, and A. Chahid, Phys. Rev. B46, 6173 (1992).

20. A.I. Krivchikov, V.G. Manzhelii, O.A. Korolyuk, B.Ya. Gorodilov, and O.O. Romantsova, Phys. Chem. Chem. Phys. 7, 728 (2005); A.I. Krivchikov, B.Ya. Gorodilov, and O.A. Korolyuk, Instrum. Exp. Techn. 48, 417 (2005).

21. A.I. Krivchikov, A.N. Yushchenko, O.A. Korolyuk, F.J. Bermejo, C. Cabrillo, and M.A. Gonzalez, Phys. Rev. B75, 214204 (2007).

22. V.A. Konstantinov, V.G. Manzhelii, M.A. Strzhemechny, and S.A. Smirnov, Fiz. Nizk. Temp. 14, 90 (1988) [Low Temp. Phys. 14, 48 (1988)].

23. A. Jagannathan, R. Orbach, and O. Entin-Wohlman, Phys. Rev. B39, 13465 (1989).

24. H. Boettger and Th. Damker, Phys. Rev. B50, 12509 (1994); T. Damker, H. Böttger, and V.V. Bryksin, Phys. Rev. B59, 8626 (1999).

25. Philip B. Allen and Joseph L. Feldman, Phys. Rev. B48, 12581 (1993).

26. Joseph L. Feldman, Mark D. Kluge, Philip B. Allen, and Frederick Wooten, Phys. Rev. B48, 12589 (1993).

27. P.B. Allen, J.L. Feldman, J. Fabian, and F. Wooten, Phil. Mag. B79, 1715 (1999).

28. A.J.H. McGaughey and M. Kaviany, Int. J. Heat Mass Transf. 47, 1783 (2004).

29. Jerzy Bodzenta, Chaos Solitons Fractals 10, 2087 (1999).

30. U. Buchenau, Yu.M. Galperin, V.L. Gurevich, D.A. Parshin, M.A. Ramos, and H.R. Schober, Phys. Rev. B46, 2798 (1992); A. Ramos and U. Buchenau, Phys. Rev. B55, 5749 (1997); D.A. Parshin, Phys. Scr. 49A, 180 (1993).

31. D.G. Cahill and R.O. Pohl, Ann. Rev. Phys. Chem. 39, 93 (1988); D.G. Cahill and R.O. Pohl, Phys. Rev. B35, 4067 (1987); D.G. Cahill, S.K. Watson, and R.O. Pohl, Phys. Rev. B46, 6131 (1992).

32. V.A. Konstantinov, V.G. Manzhelii, V.P. Revyakin, and V.V. Sagan, Fiz. Nizk. Temp. 31, 553 (2005) [Low Temp. Phys. 31, 419 (2005)]; V.A. Konstantinov, V.G. Manzhelii, V.P. Revyakin, and V.V. Sagan, Fiz. Nizk. Temp. 32, 1414 (2006) [Low Temp. Phys. 32, 1076 (2006)]; V.G. Manzhelii, V.A. Konstantinov, V.P. Revyakin, V.V. Sagan, and O.I. Pursky, J. Phys.: Cond. Matt. 18, 9901 (2006).

33. A. Srinivasan, F.J. Bermejo, and A.De Bernabe, Mol. Phys. 87, 1439 (1996).

34. V.A. Konstantinov, E.S. Orel, V.P. Revyakin, Fiz. Nizk. Temp. 29, 1007 (2003) [Low Temp. Phys. 29, 759 (2003)]; V.A. Konstantinov, Fiz. Nizk. Temp. 29, 567 (2003) [Low Temp. Phys. 29, 422 (2003)]. 